\title{
Measuring and Modeling the Bidirectional Reflection Distribution Function of Space Object's Surface Material
}

\author{
Peng $\mathrm{LI}^{1, \mathrm{a}}$, Zhi $\mathrm{LI}^{2, \mathrm{~b}}$ and Can XU ${ }^{3, \mathrm{c}}$ \\ ${ }^{1}$ Department of Graduate Management, Equipment Academy, Beijing 101416, China; \\ ${ }^{2}$ Equipment Academy, Beijing 101416, China; \\ ${ }^{3}$ Department of Space Equipment, Equipment Academy, Beijing 101416, China; \\ a693078757@qq.com, 'lizhizys@263.com, c452394317@qq.com
}

Keywords: bidirectional reflection distribution function (BRDF), relative measurement, space object, five-parametric model, the simulated annealing algorithm

\begin{abstract}
Bidirectional reflection distribution function (BRDF) is broadly used in many fields, such as physics, remote sensing, computer graphics and scattering in optical band and microwave band. The BRDF measurement instrument used to obtain raw measurement data is presented firstly. The BRDF data of two typical space object's surface materials (yellow and silver foil) are measured. Measurement results show that the scattering angle corresponding to the maximum value of BRDF curve is generally in the specular reflection direction; the other BRDF values decrease quickly as scattering angle deviate from the specular reflection direction. The optimum parameter of the five-parameter BRDF model are retrieved based on simulated annealing algorithm and the root mean square error is less than $6 \%$. The feasibility and reliability of the modeling are verifed by comparing the data calculated by the five-parametric model and the data measured originally. The measuring and modeling results could provide reference for space object detection and identification.
\end{abstract}

\section{Introduction}

Bidirectional reflection distribution function (BRDF) is used to describe the scattering characteristic of the surface of object material; it is mainly determined by the surface roughness, dielectric constant, exposure or reflective wavelength and polarization etc[1]. It is a fundamental radiometric concept and plays a significant role in Earth remote sensing, heat translation, and so on[2]. It takes an incoming light direction and outgoing direction that both defined with respect to the surface normal as input parameters, returns the ration of reflected radiance exiting along reflection direction to the irradiance that illuminate on the material surface.

The analytical models of BRDF are divided into physical models and statistical models. Physical models are based on a certain physical principle, such as Davies model[3], Maxwell Beard model[4]; while statistical models are based on a large number of experimental data and the parameters of this kind of models are not practical such as Torrance Sparrow model[5], five-parameter model[6] and etc. As statistical models don't need information about optical and roughness parameters of the material surface, statistical models are more intuitive and practical.

Tec Light company in France produces a new BRDF measurement instrument with high degree of automation and accuracy, namely REFLET 180S. In this paper, the BRDF of two typical space object's surface materials (yellow and silver foil) are measured by REFLET 180S. Measurement results show that BRDF of these two materials are strongly related to reflective angle and the peak usually deviates $0.2^{\circ} \sim 0.3^{\circ}$ from specular direction.

\section{Methodology}

BRDF is a function describing behavior of reflection of light on material surface, can be applied to describe reflection characteristic [7]. BRDF measurement methods are divided into absolute 
measurement and relative measurement. A relative measurement method is adopted to measure BRDF data of two space object's surface materials. Polytetrafluoroethylene (F4) is used as reference standard board in REFLET 180S, whose hemispherical reflectance is ${ }^{\rho}$ calibrated by United States National Academy of Sciences. The BRDF of F4 is $\rho / \pi$. The geometry and notations for BRDF are shown in Figure 1. The mathematical expression of BRDF $f_{r}$ is:

$$
f_{r}\left(\theta_{i}, \varphi_{i} ; \theta, \varphi_{r}\right)=\frac{L_{r}\left(\theta_{i}, \varphi_{i} ; \theta_{r}, \varphi_{r}\right) \cos 30^{\circ}}{L_{b}\left(30^{\circ}, \varphi_{i} ; \theta_{r}, \varphi_{r}\right) \cos \theta_{r}} \cdot \frac{\rho}{\pi}
$$

where $\theta$ is the zenith angle, $\varphi$ is the azimuthal angel, the subscripts $i$ and $r$ represent incident and reflected direction; $L_{r}\left(\theta_{i}, \varphi_{i} ; \theta_{r}, \varphi_{r}\right)$ is the reflected radiance in unit $\mathrm{W} / \mathrm{m}^{2} \cdot s r$ when the light illuminate the material at the incident angle $\left(\theta_{i}, \varphi_{i}\right) ; L_{b}\left(30^{\circ}, \varphi_{i} ; \theta_{r}, \varphi_{r}\right)$ is the reflected radiance in unit $\mathrm{W} / \mathrm{m}^{2} \cdot s r$ when the light illuminate the reference standard board at the incident angle $\left(30^{\circ}, \varphi_{i}\right)$. When the material is isotropic, the incident azimuthal angle can be assumed as 0 , then the azimuthal angel is $\varphi=\varphi_{r}-\varphi_{i}=\varphi_{r}$

From formula (1) we can see that, in order to obtain BRDF values, only a set of measured data on the reference standard board at the incident angle of $30^{\circ}$ is needed and this is the principle of REFLECT 180s we used in the paper.

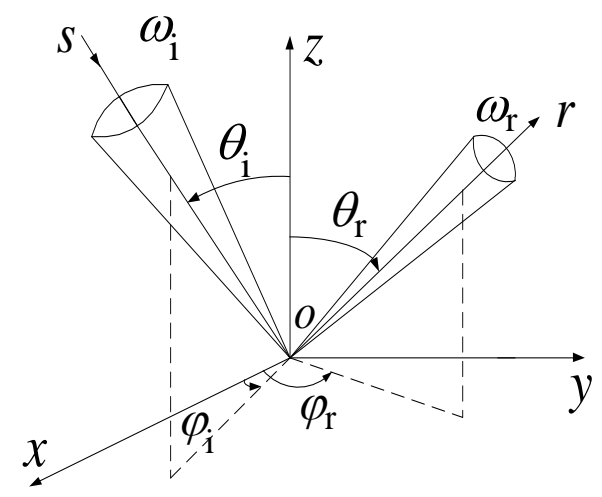

Fig. 1 Geometry and notations in BRDF definition

\section{Measurements}

\subsection{The measurement instrument- REFLET 180S}

REFLET 180S is made by Light Tec in France. The optical module consists of two symmetricrotating optical systems (illumination system and detection system) focused on the same point on the sample surface. A darkroom protects the optical bench from stray light. The instrument is transportable thanks to the removable handles locate on the optical bench. The measurement instrument- REFLET 180S is shown in Figure 2. The main characteristics of REFLET 180S includes:

-Optical bench: illumination module, comprising one optical system with 2 diaphragms and one motorized 1-axis goniometer (control the incident elevation angle $\theta_{i}, 0^{\circ} \leq \theta_{i} \leq 180^{\circ}$ ); detection module, comprising one interchangeable optical bloc and one motorized 2-axis goniometer (control the reflection elevation angle $\theta_{d}$ and azimuth angle $\varphi_{d},-90^{\circ} \leq \theta_{d} \leq 90^{\circ}$ and $\varphi_{d},-90^{\circ} \leq \varphi_{d} \leq 90^{\circ}$ )

-Electronic cupboard: motion controllers and power supply for the goniometer motors and the detectors: one 4-photodiode "Intensity” type detector (standard), one 2-photodiode 'Infra Red" type detector and one spectrograph (option) detector. The desired detector is selected using a fiber optics located on the front panel of the cupboard.

- Light box: a 100W tungsten Halogen lamp with the wavelength ranges from 400 1800nm. The lamp can be changed by switching the trap at the rear side of the box.

-Processing computer: the entire measurement process is computer automated and saving time. 


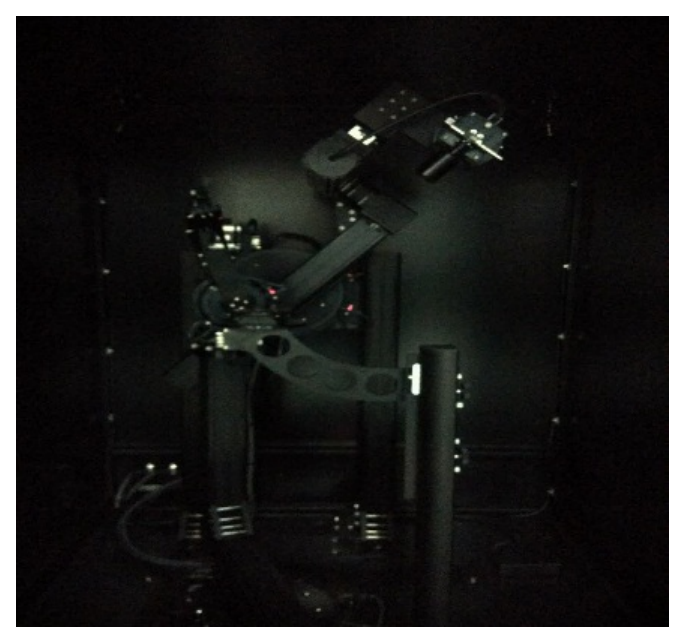

(a) BRDF measurement systems in the darkroom

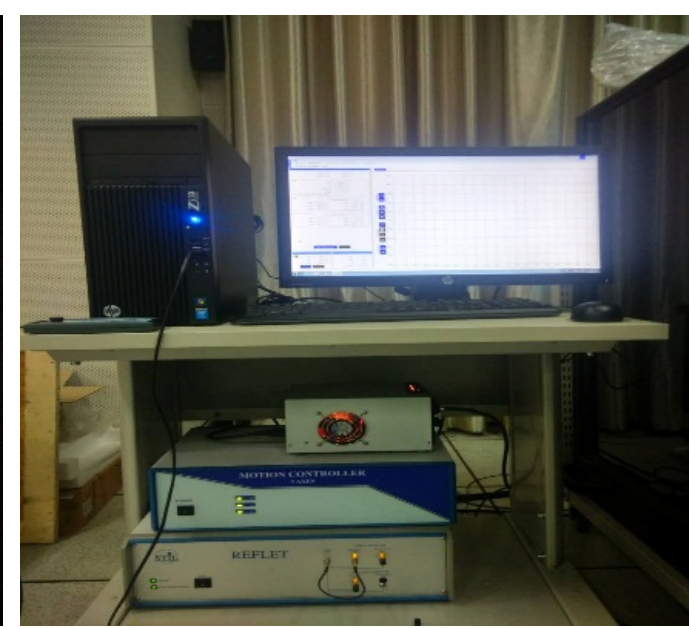

(b) a computer with dedicated boards and software

Fig. 2 The BRDF measurement instrument-REFLET 180S

\subsection{BRDF measurement}

BRDF data of typical space object's surface materials (yellow and silver foil) are measured using REFLET 180S. The incident zenith angle ranges from $15^{\circ}$ to $75^{\circ}$ at interval of $15^{\circ}$; the azimuth angle ranges from $180^{\circ}$ to $200^{\circ}$ at interval of $1^{\circ}$. Because the specular reflection phenomenon of space object's surface coating material is obvious, in order to get enough measurement data near mirror reflection direction, the reflected zenith angle ranges from $-90^{\circ}$ to $90^{\circ}$ at interval of $0.1^{\circ}$. Taking device limitations into account, when detector and light source locate at the same direction, the detector will block some light and result in some data losses. Two space object's surface materials measured in the paper are shown in Figure 3.

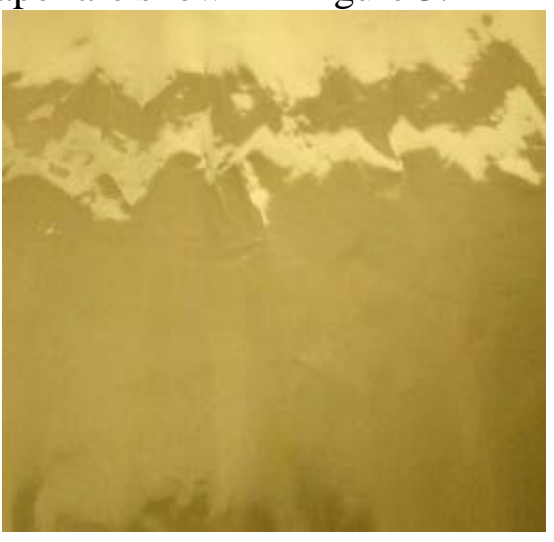

(a) yellow foil

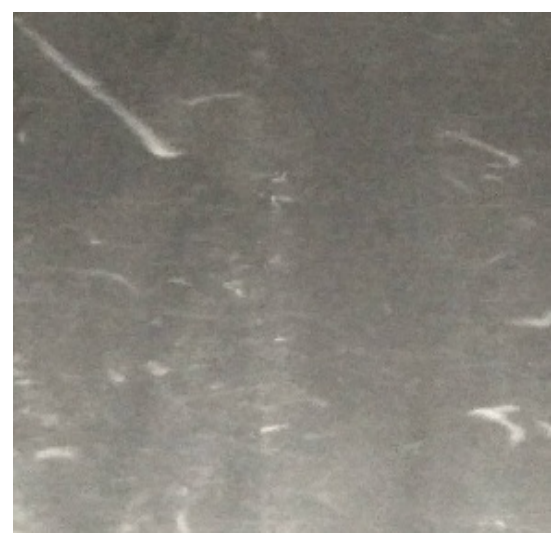

(b) silver foil

Fig. 3 The space object's surface materials

\section{Measurements result and data analysis}

\subsection{Measurements result}

The BRDF of the space object's surface materials (yellow and silver foil) are measured by REFLET 180S. Some BRDF measurements with different incident angle and reflection angle are shown in Figure 4 Figure 5. 


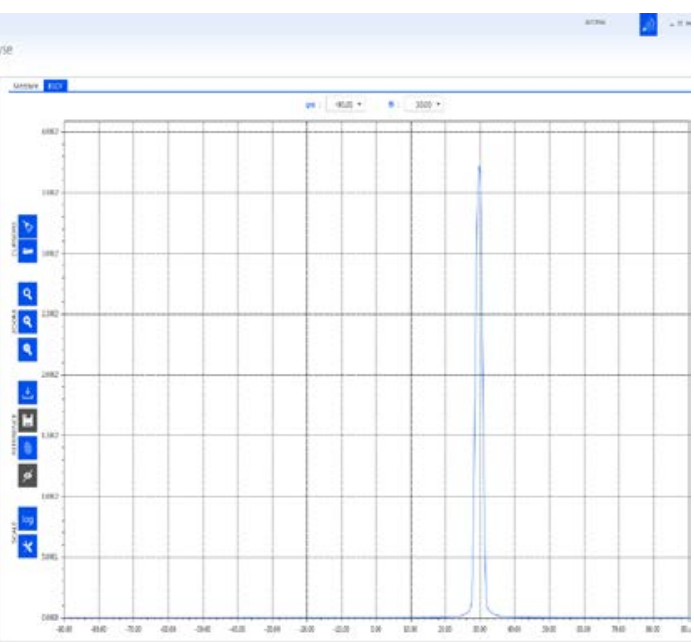

(a) incident angle of $30^{\circ}$

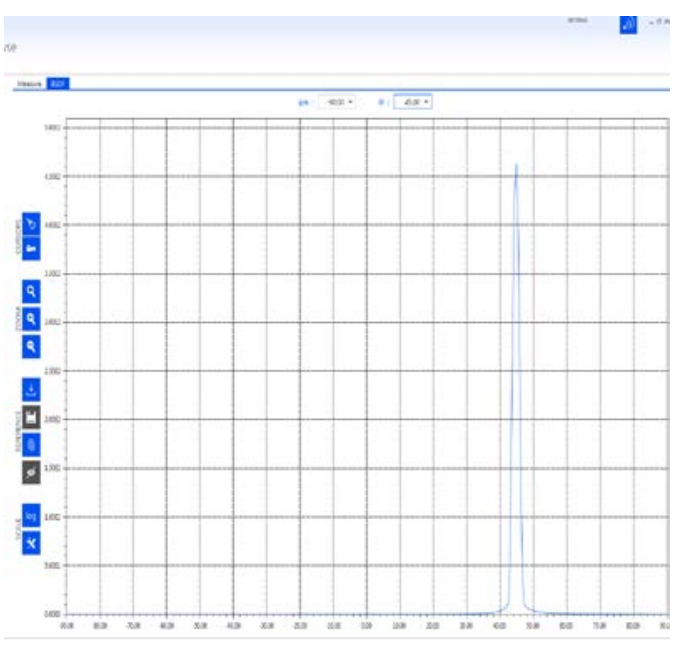

(b) incident angle of $45^{\circ}$

Fig. 4 Measured BRDF for yellow foil

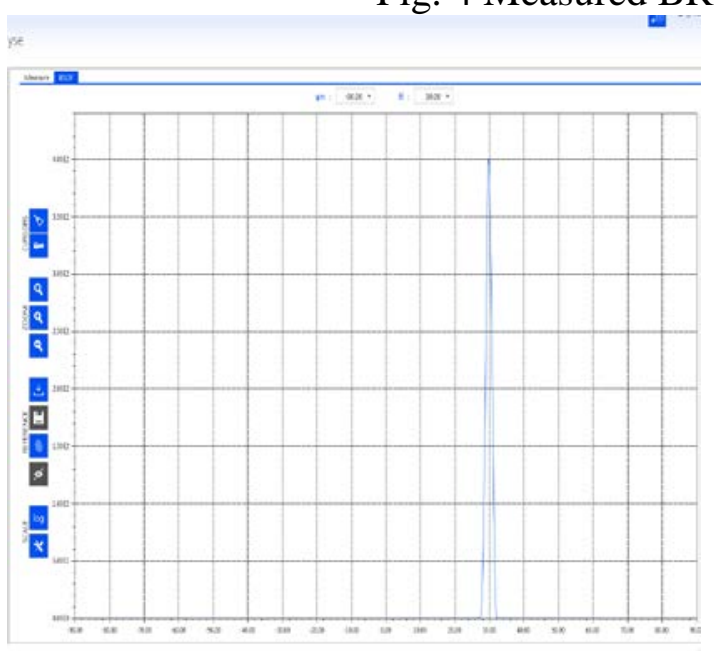

(a) incident angle of $30^{\circ}$

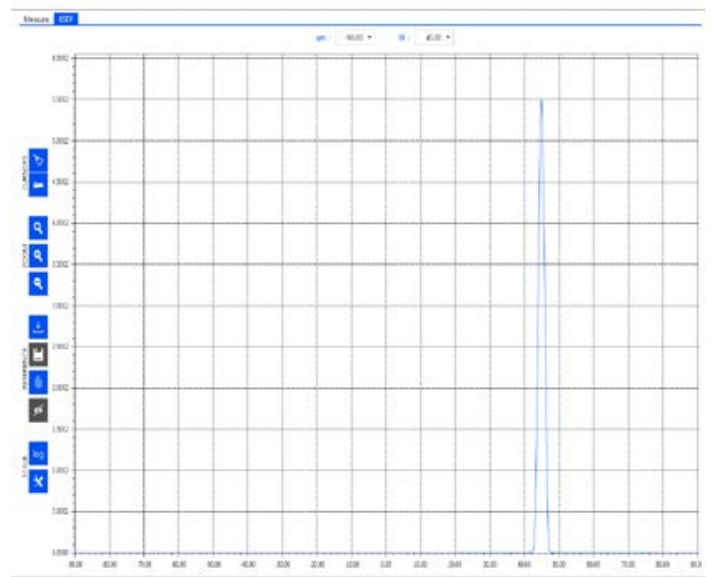

(b) incident angle of $45^{\circ}$

Fig. 5 Measured BRDF for silver foil

\subsection{Measurement Analysis}

It is obvious that BRDF is strongly related to reflective angle and this is provided by our measurement result. From Fig 4 Fig 5, a non-specular peak near the reflection angle appeared and the peak usually deviates $0.2^{\circ} \sim 0.3^{\circ}$ from specular direction. BRDF values decrease significantly when reflection angle deviate from the specular reflection direction.

For the two materials being measured, peaks of BRDF at incident zenith angle of $45^{\circ}$ are larger than the peak at the incident angel of $30^{\circ}$. That means that the value of BRDF becomes larger with the increasing of incident zenith angle.

\section{3 five-parameter model}

The mathematical expression of five-parameter model is:

$$
f_{r}=k_{b} \frac{k_{r}^{2} \cos a}{1+\left(k_{r}^{2}-1\right) \cos a} G\left(\theta_{i}, \theta_{r}, \varphi_{r}\right) \cdot \frac{\exp \left[b(1-\cos \gamma)^{a}\right]}{\cos \theta_{i} \cos \theta_{r}}+\frac{k_{d}}{\cos \theta_{i}}
$$

where $\theta_{i}$ is the incident zenith angle, $\theta_{r}$ is the reflected zenith angle , $\varphi_{r}$ is the azimuth angel; $\alpha$ is the angle between normal direction $\stackrel{\mathbf{q}}{n}$ and axis $z$ on the micro face, $\gamma$ is the incident angle in local coordinate system on the micro face; $k_{b}, k_{r}, b, a, k_{d}$ are five parameters to be calculated; each parameter has be specified explicitly in literature[6].

The optimum values of the five parameters are the values that could make the normalized root mean square error (NRMSE) of fitting experimental data minimum. The mathematical expression of objective function is: 


$$
E(x)=\frac{\sum_{\theta_{1}} \sum_{\theta_{r}} g_{1}\left(\theta_{i}\right) g_{2}\left(\theta_{r}\right)\left[f_{r}\left(\theta_{i}, \theta_{r}, \varphi_{r}\right) \cos \theta_{r}-f_{r}^{0}\left(\theta_{i}, \theta_{r}, \varphi_{r}\right) \cos \theta_{r}\right]^{2}}{\sum_{\theta_{i}} \sum_{\theta_{r}} g_{1}\left(\theta_{i}\right) \mathrm{g}_{2}\left(\theta_{r}\right)\left[f_{r}^{0}\left(\theta_{i}, \theta_{r}, \varphi_{r}\right) \cos \theta_{r}\right]^{2}}
$$

where $x=\left[k_{b}, k_{r}, b, a, k_{d}\right]^{T}$ is the column vector of the parameter model; $g_{1}\left(\theta_{i}\right) g_{2}\left(\theta_{\mathrm{r}}\right)$ is a weight function that the value is 1 in equidistant measurement. As the expression of five-parameter model is complex and the objective function is nonlinear, the optimization function is often used to solve the question. In this paper, the optimum parameters of the model are retrieved by simulated annealing algorithm. The five parameters fitted out are shown in Table 1.

Table 1 The five-parameter model result of surface material

\begin{tabular}{ccccccc}
\hline Material & $k_{b}$ & $k_{r}$ & $b$ & $a$ & $k_{d}$ & $E(x) /(\%)$ \\
\hline Yellow foil & 323.173 & 0.00419 & 1.48651 & 0.53556 & 0.02357 & 5.36 \\
Silver foil & 326.995 & 0.00363 & 2.63009 & 0.71188 & 0.03250 & 5.57 \\
\hline
\end{tabular}

In order to indicate the fitting results of the five-parameter model, fitting result and measurements are given out in Figure 6 Figure 7 at incident zenith $45^{\circ}$ angle.

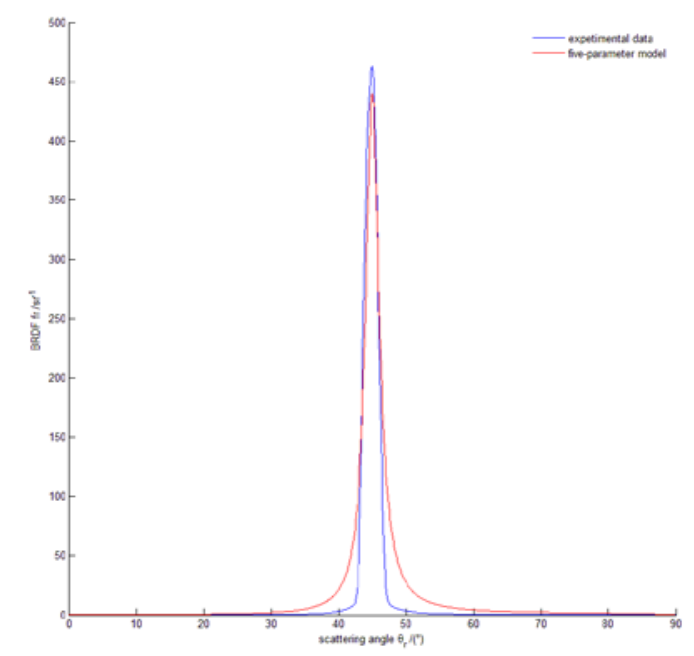

Fig. 6 The fitting results of yellow foil

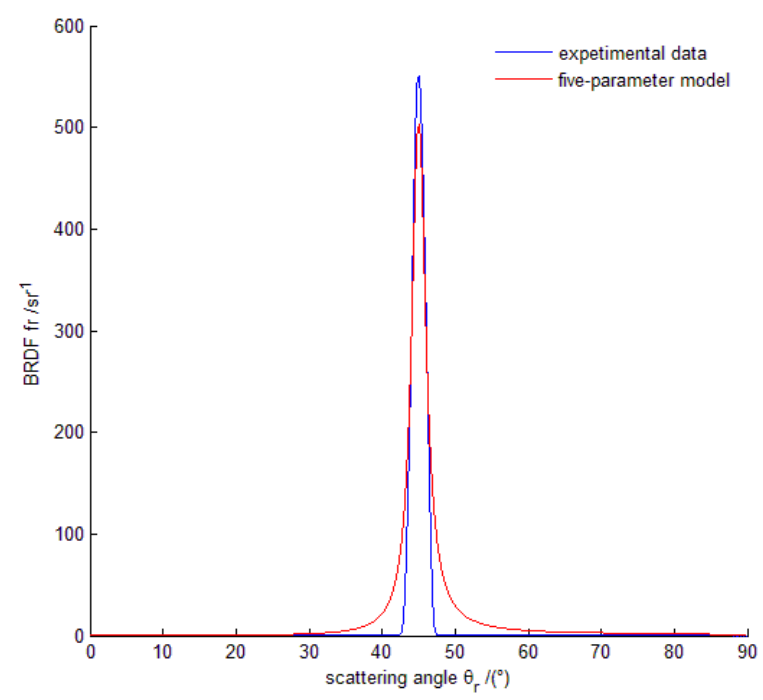

Fig. 7 The fitting results of silver foil

In Figure 6, it can be seen that the peak of experimental data is slightly larger than fitting result and when reflection angle deviate from $45^{\circ}$, fitting result is becoming larger than experimental data. And 
the root mean square error is controlled within 6\%. It indicate that five-parameter model performed well with experimental measurement data. The same conclusion can be drawn from Figure 7.

\section{Summary}

BRDF data of two kinds of typical space object's surface coating materials (yellow and silver foil) are measured by REFLET 180S and the BRDF characteristics with the scattering angle is analyzed. Based on five-parameter model of BRDF, the optimum parameter of the model are retrieved by simulated annealing algorithm and the root mean square error is less than $6 \%$. By comparing fitting results with the experimental data, it is concluded that the five-parameter model is feasible and reliable. Research emphases in the future is to establish a complete BRDF database for space object's surface material and apply it to space object detection and recognition.

\section{References}

[1]. YANG Yufeng, WU Zhensen, CAO Yunhua. Optical constants deduction and scattering BRDF computation of alloy aluminum surface in near infrared waveband, J. Acta Optica Sinica. Vol.32 (2011) No. 2, p. 262-267. (in Chinese)

[2]. Ward, Gregory J. Measuring and modeling anisotropic reflection. J. ACM SIGGRAPH Computer Graphics, Vol. 26 (1992) No. 2, p. 265-272.

[3]. SHEN, Y J, Zhang Z M, Tsai B K. Bidirectional reflectance distribution function of rough silicon wafers, J. International Journal of Thermophysics. Vol. 22 (2001) No. 4, p. 1311-1326.

[4]. Maxwell J R, Beard J, Weiner S, et al. Bidirectional Reflectance Model Validation and Utilization. R. ENVIRONMENTAL RESEARCH INST OF MICHIGANANN ARBOR INFRARED AND OPTICS DIV, (1973).

[5]. Torrance, Kenneth E., and Ephraim M. Sparrow. Theory for off-specular reflection from roughened surfaces. JOSA . Vol. 57 (1967) No. 9, p. 1105-1112.

[6]. WU Zhensen, XIE Don-hui, XIE Pinhua, et al. Modeling reflectance function from rough surface and algorithms, J. Acta Optica Sinica, Vol. 22 (2002) No. 8, p. 897-901. (in Chinese)

[7]. Zhang W, Wang F, Wang Z, et al. Measuring of spectral BRDF usingopticspectrometer[C]//Proc. of SPIE Vol. 7658: 76582P-1. 